\title{
CONCENTRATION FREQUENCY DEPENDENCE OF SHORT RANGE CHAIN FLUCTUATIONS OBSERVED IN CONCENTRATED POLYMER SOLUTIONS FROM NUCLEAR MAGNETIC RELAXATION RATES
}

\author{
J. P. COHEN-ADDAD and J. P. MESSA \\ Laboratoire de Spectrométrie Physique (*), Université Scientifique et Médicale de Grenoble, \\ B.P. 53, 38041 Grenoble Cedex, France
}

(Reçu le 26 avril 1976, accepté le 14 mai 1976)

\begin{abstract}
Résumé. - Les modes relaxationnels des fluctuations de chaînes dans les solutions concentrées de polymères sont observés à partir de la relaxation magnétique des protons. Les vitesses de relaxation spin-réseau et dans le référentiel tournant, mesurées sur les chaînes de polyisobutylène, sont indépendantes du poids moléculaire entre $5 \times 10^{4}$ et $10^{6}$. Elles sont gouvernées par un mécanisme attribué aux modes élevés des fluctuations de chaînes; ceux-ci sont contrôlés par la concentration de solvant par l'intermédiaire du volume libre statique introduit par Williams - Landél et Ferry.
\end{abstract}

\begin{abstract}
Relaxational modes of short range chain fluctuations in concentrated polymer solutions are investigated by studying the proton magnetic relaxation rates. The relaxation rate in the rotating frame and the spin-lattice relaxation rate observed in polyisobutylene chains are found to be independent of the molecular weight in the range $5 \times 10^{4}$ to $10^{6}$. They are governed by a mechanism which is ascribed to high frequency chain fluctuations controlled by the solvent concentration through the static free volume introduced into the Williams-Landel-Ferry equation. Free volume parameters are evaluated.
\end{abstract}

1. Introduction. - Dynamical scaling laws have been recently shown to apply to the description of the concentration dependence of the low frequency behaviour of semi-dilute polymer systems, observed at definite wave-length from light scattering or ultrasonic measurements [1]; the polymer concentration appears as a capital thermodynamic parameter in such a description. On the contrary, high modes of the chain relaxation spectrum of concentrated polymer solutions are more arduous to describe. Consequently, in the experimental determination of the concentration-frequency dependence of segmental chain motions, the high frequency range has been left relatively unexplored. The use of reduced variables is the approach which has been currently considered $[2,3]$. Most nuclear magnetic relaxation (NMR) processes observed on polymer systems are known to result from dipolar spin coupling fluctuations. This short range spin interaction permits one to probe segmental chain motions on a short space scale corresponding to high relaxational modes. To our knowledge, no quantitative investigation has been made of the solvent concentration dependence of either the spin-lattice relaxation rate or the relaxation

$\left(^{*}\right)$ Associé au C.N.R.S. rate in the rotating frame, observed on concentrated polymer solutions.

The temperature frequency dependence of spinlattice relaxation rates observed for some pure molten polymers has suggested that the rate, $W$, of the relevant molecular motions is sensitive to local volume fluctuations [4]. As in ordinary liquids [5, 6], these are assumed to be controlled by the temperature through the static free volume, $f$, introduced in the Williams-Landel-Ferry (WLF) equation :

$$
W_{1} W_{2}^{-1} \propto \exp \left(f_{1}^{-1}-f_{2}^{-1}\right)
$$

where the indices 1 and 2 refer to two different states of the system [7]. The purpose of the present letter is to show that high relaxational modes of segmental chain motions also exhibit a solvent concentration dependence through the static free volume, $f$. We report experimental features obtained from relaxation rates observed for highly concentrated polyisobutylene (PIB) chain solutions. In order to have a rigorous frequency analysis, we considered two types of relaxation processes which are known to correspond to a strictly locked observation frequency : the relaxation in the rotating frame and the spinlattice relaxation. Experiments on PIB were performed 
on samples corresponding to three different average molecular weights $\left(10^{6}, 10^{5}\right.$ and $5 \times 10^{4}$, respectively) in order to probe any possible chain length effect. Two types of good solvents were used $\left(\mathrm{CDCl}_{3}\right.$ and $\mathrm{CS}_{2}$ ) to eliminate any spin concentration effects. The investigation frequency of the relaxation process in the rotating frame was $7 \times 10^{3} \mathrm{~Hz}$. The time evolution of the transverse magnetization in the rotating frame was found to be described according to an exponential function in the whole concentration range. Furthermore, the longitudinal component approached its equilibrium value according to an exponential function.

2. The free volume law. - The concentration dependence of the proton relaxation rate in the rotating frame observed on PIB samples corresponding to three different chain molecular weights, is shown in figure 1 , using a representation corresponding to the WLF equation :

$T_{1} \rho\left(v_{1}^{0}\right) / T_{1} \rho\left(v_{1}\right)=\exp \left\{\left(v_{1}-v_{1}^{0}\right) /\left(a+b\left(v_{1}-v_{1}^{0}\right)\right)\right\}$,

(1)

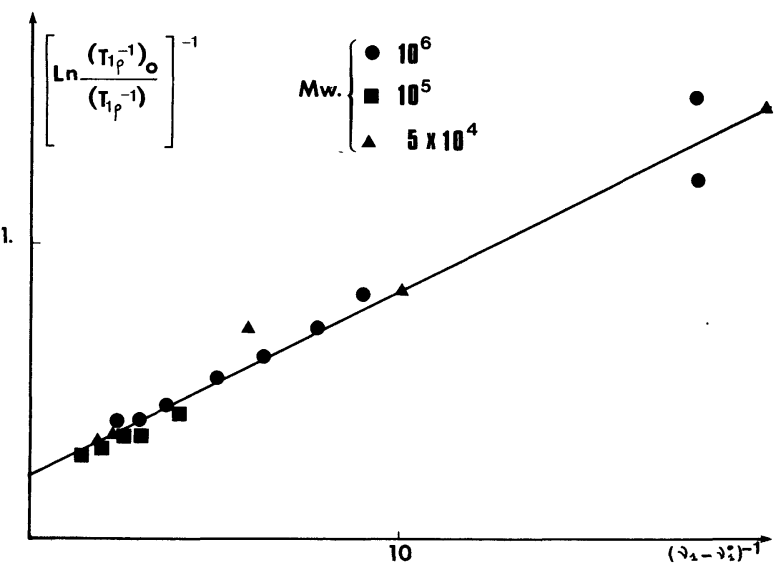

Fig. 1. - Variation of the relaxation rate in the rotating frame as a solvent concentration function $\left(\mathrm{CDCl}_{3}\right)$. The WLF representation is used.

$v_{1}$ is the solvent molar concentration and $v_{1}^{0}$ is the lowest observed solvent concentration ;

$$
a=\left(f\left(v_{1}^{0}\right)\right)^{2} /\left(f_{1}-f_{2}\right)=0.062 ;
$$

$f_{1}$ and $f_{2}$ are the free volume contributions of the pure solvent and polymer, respectively;

$$
b=f\left(v_{1}^{0}\right)=f_{2}+v_{1}^{0}\left(f_{1}-f_{2}\right)=0.22 ;
$$

$f_{2}=0.1$ and $f_{1}=0.8$ for $\mathrm{CDCl}_{3}$. These values must be compared with $f_{2}^{1}=0.08$ and $f_{1}^{1}=0.3$ derived from other measurements at lower frequencies. A carefull analysis of experimental results which is not reported here, permits one to eliminate other types of solvent concentration dependences. The relaxation rate is insensitive to molecular weight, at room tem- perature. The direct representation of the concentration dependence of $T_{1 \mathrm{p}}$ is shown in figure 2 . The concentration dependence of the spin-lattice relaxation rate observed on PIB samples at 10 and $32 \mathrm{MHz}$, at room temperature, is shown in figure 3. The following analysis of results is now given.

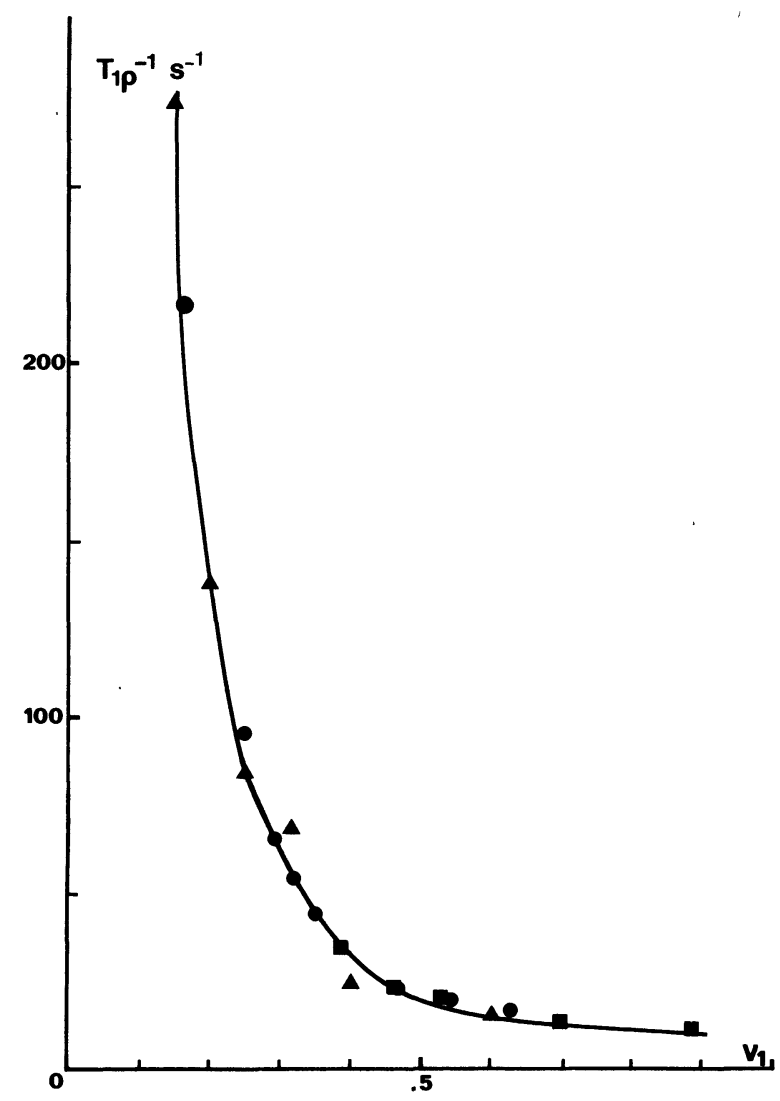

FIG. 2. - Direct representation of the concentration dependence of $T_{1 \mathrm{p}}$. Key to points same as in figure 1 .

In the ref. [8], attention was focused on the temperature dependence of the proton spin-lattice relaxation rate observed for pure polyisobutylene chains at two radiofrequencies, 30 and $50 \mathrm{MHz}$. Two maxima were observed in the temperature interval $170 \mathrm{~K}$ to $400 \mathrm{~K}$. The low temperature maximum $(250 \mathrm{~K})$ was ascribed to three-fold rotation of the methyl groups about the $\mathrm{C}-\mathrm{C}$ bond. The high temperature maximum (332 and $339 \mathrm{~K}$ at 30 and $50 \mathrm{MHz}$, respectively) was ascribed to fluctuations of chain segments. Also, the spin-lattice relaxation rate was found to be insensitive to molecular weight for $M_{\mathrm{W}}>10^{4}$, in the temperature range $T>273 \mathrm{~K}$, at $50 \mathrm{MHz}$.

3. The shift factor. - The observation of one maxımum in tigure 3 on the one hand and the concentration-frequency dependence of the maxima on the other hand are qualitatively what one would expect if the relevant molecular rate governing the spinlattice relaxation process is assumed to be an increasing function of solvent concentration. The maximum 


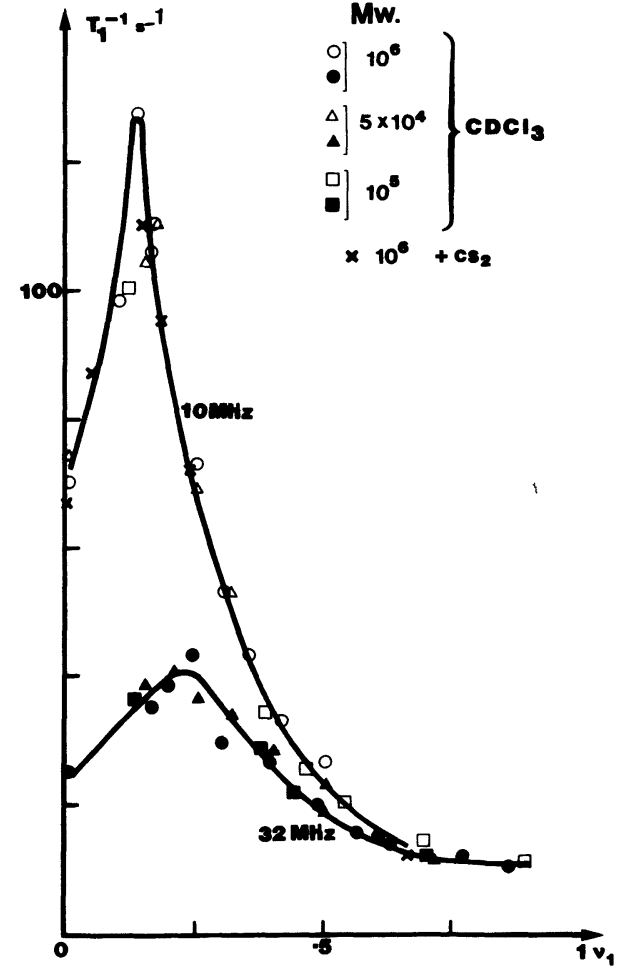

Fig. 3. - Variation of the spin-lattice relaxation rate as a solvent concentration function $\left(\mathrm{CDCl}_{3}\right)$. Relaxation rates of PIB chains in $\mathrm{CS}_{2}$ are also measured for comparison with $\mathrm{CDCl}_{3}$.

amplitudes of spin-lattice relaxation rates were also measured as a function of temperature for a few pure polymer systems at several frequencies. These rates exhibit a reasonable linear dependence with respect to $\omega_{0}^{-1} ; \omega_{0}$ is the Larmor frequency. The same property can be seen from figure 3 . The temperature dependence reported in the curves given in ref. [8] illustrates the same property. The ratio of the two maxima corresponding to 30 and $50 \mathrm{MHz}$, respectively, is found to be $1.7 \simeq 50 / 30$. The concentration frequency dependence of the relaxation rates is therefore expected to be described by the homogeneous function :

$$
\omega_{0} T_{1}^{-1}=\bar{D}^{2} F\left(\omega_{0} / W\left(v_{1}, T\right)\right)
$$

where $\bar{D}^{2}$ is the average dipolar spin-coupling, $W$ is a fluctuation characteristic frequency, depending upon $v_{1}$ and the temperature $T$. From the description given by eq. (2), the curves $\omega_{0} T^{-1}\left(\omega_{0}, W\right)$ should be derived from each other from a simple shift along the solvent concentration axis. The shift factor is defined as $W\left(v_{1}, T\right) / \omega_{0}=$ const. From the concentration dependence of the relaxation rate in the rotating frame, it is now supposed that $W\left(v_{1}\right)$ obeys the free volume law (1). The two curves shown in figure 3 should therefore be shifted from each other by the above simple factor. This property is clearly illustrated in figure 4 where the curve $W T_{1}^{-1}$ drawn as a $W$ function is shown at 10 and $32 \mathrm{MHz}$. The agreement between experimental points and the shift factor is within less than 10 per cent of uncertainty. Consequently, both magnetic relaxation processes can be considered to be governed by the same molecular relaxation rate $W$. They are insensitive to molecular weight. They obey a free volume law. The spin-lattice relaxation rate exhibits a maximum amplitude corresponding to a molecular rate $W \simeq 10^{8} \mathrm{rad} . \mathrm{s}^{-1}$. The $W$ values shown in figure 4 are calculated with arbitrary units and they are derived from the free volume law (1).

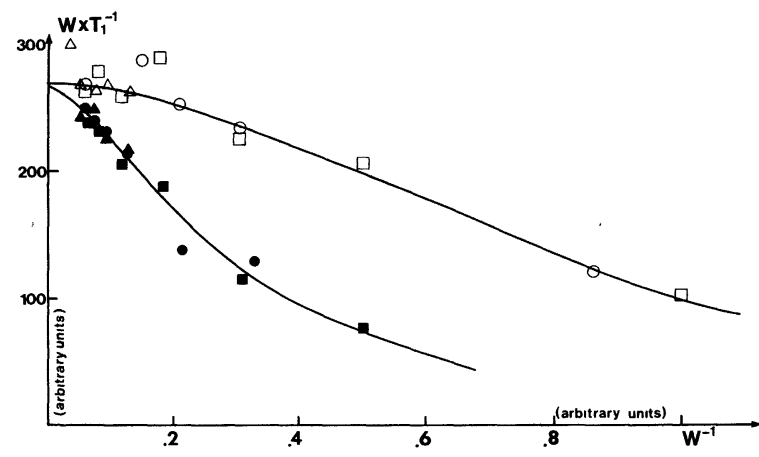

FIG. 4. - Representation of $T_{1}^{-1} \exp \left\{\left(v_{1}-v_{1}^{0}\right) / a+b\left(v_{1}-v_{1}^{0}\right)\right\}$ derived from experimental points. Key to points same as in figure 2 . The frequency shift is clearly perceived in this figure.

These features must be compared with results obtained from viscoelastic measurements performed on PIB chains. In semi-dilute polymer solutions chain motions must be described according to a Zimm model [9] in order to take free draining effects into consideration; whereas in concentrated solutions, viscous drag effects of chain segments can be ignored and the Rouse formulation of chain motion can be used [10]. Accordingly, in the case of concentrated solutions, relaxational modes depend explicitly on the chain segment mobility, $\zeta$. This is usually supposed to be controlled by the solvent concentration through the static free volume, $f$. The corresponding concentration dependence of the complex shear modulus is entirely dominated by that of $\zeta$. In other words, relaxational modes which are known to reflect the pseudogel rigidity modulus resulting from the presence of entanglements, must decrease with solvent addition [1] This effect is however screened by the concentration dependence of $\zeta:$ a free volume law is actually observed from low frequency viscoelastic measurements. Although the Rouse model must be given a modified treatment to calculate high relaxational modes [3], these depend roughly on the chain segment mobility $\zeta$; and they should also exhibit a free volume law. Furthermore, the shear loss modulus $G^{\prime \prime}(\omega)$, observed for pure molten PIB chains [11], has been predicted to exhibit a maximum amplitude at $\omega \simeq 10^{8} \mathrm{rad} . \mathrm{s}^{-1}$, by using reduced variables. In that case it is possible that the NMR results and viscoelastic measurements obtained from PIB chains are related to one other. 
4. Qualitative interpretation. - In an attempt to describe qualitatively the NMR results reported here, we consider the two-spin system model which has already been used to calculate the residual average dipolar spin coupling observed for molten polymers [12-15]. We assume that a proton pair is located on a bond of a freely jointed chain sub-unit. When this gaussian chain segment is submitted to an extension, $\mathbf{r}$, the local rotation of the freely jointed bond does not average the dipolar spin coupling, $\overline{\mathfrak{H}}_{\mathrm{D}}$, to zero; and within the proton pair we have $\overline{\mathfrak{H}}_{\mathrm{D}} \propto r^{2}$. The residual spin-coupling is then sensitive to entropy fluctuations of the chain which correspond to variations of the number of configurations available to the segment. The residual spin-coupling, $r^{2}$, is modulated by fluctuations of the end-to-end vector, $r$. The spin-lattice relaxation process of the two-spin system depends upon the relaxation function : $\left\langle r^{2}(0) r^{2}(t)\right\rangle$. This can be evaluated using the Rouse model; the corresponding spectral density $J(\omega)$ is shown without any difficulty to be related to the viscoelastic shear loss modulus $G(t)$ :

$$
J(\omega)=\int[G(t) / n k T]^{2 \cdot} \times \mathrm{e}^{i \omega t} \mathrm{~d} t ;
$$

$n$ is the number of polymer molecules per cc. This is obviously a rough model but it indicates that NMR and viscoelastic measurements can be related to each other.

Accordingly, from the present investigation we believe that high relaxational modes of chain fluctuations can be probed from NMR relaxation rates. These are not sensitive to the specific nature of the monomer unit. The same free volume dependence must appear through viscoelastic and NMR measurements. This result offers a qualitative justification for the use of reduced variables currently considered to explore high relaxational modes from viscoelastic measurements performed from 10 to $10^{4} \mathrm{~Hz}$. Since NMR processes probe short range segmental chain fluctuations, both the relaxation rate in the rotating frame and the spin-lattice relaxation rate are sensitive to the same high modes of the chain relaxation spectrum. This explains why the same molecular rate, $W$, is used to interpret the solvent concentration dependence of both relaxation rates. In other words, the relaxation process in the rotating frame does not probe low frequency chain motions.

Analogous results were observed from relaxation rates measured on cis-1,4-polybutadiene chains. The lack of space does not permit the inclusion of the relevant results. Observation of the temperature and concentration dependences of the spin relaxation rates should shed more light on the details discussed above.

\section{References}

[1] De Gennes, P. G. (to be published in Macromolecules).

[2] Ferry, J. D., Viscoelastic properties of Polymers (Wiley, New York) 1970.

[3] Marvin, R. S. and Oser, H., J. Res. Nat. Bur.Stand. 66B - (1962) 171

[4] Slichter, W. P., J. Polym. Sci. 14 (1966) 33.

[5] Bartoli, F. J. and Litovitz, T. A., J. Chem. Phys. 56 (1972) 413

[6] Vanderhart, D. L., J. Chem. Phys. 60 (1974) 1858.

[7] Williams, M. C., Lander, R. F. and Ferry, J. D., J. Am. Chem. Soc. 77 (1955) 3701.

[8] Slichter, W. P. and Davis, D. D., J. Appl. Phys. 35 (1964) 3103.
[9] Zimm, B. H., J. Chem. Phys. 24 (1956) 269.

[10] Rouse, P. E., J. Chem. Phys. 2 (1953) 1272.

[11] OSER, H. and MARVIN, R. S., J. Res. Nat. Bur. Stand. 67B (1963) 87

[12] Cohen-Addad, J. P. and Vogin, R., Phys. Rev. Lett. 33 (1974) 940.

[13] Cohen-Addad, J. P. and Roby, C., J. Chem. Phys. 63 (1975) 3095.

[14] Cohen-Addad, J. P., J. Chem. Phys. 63 (1975) 4880.

[15] Cohen-Addad, J. P., J. Chem. Phys. (to be published in the issue of April 1976).

Commission paritaire $\mathrm{N}^{\circ} \mathbf{2 6 . 7 4 1}$ 